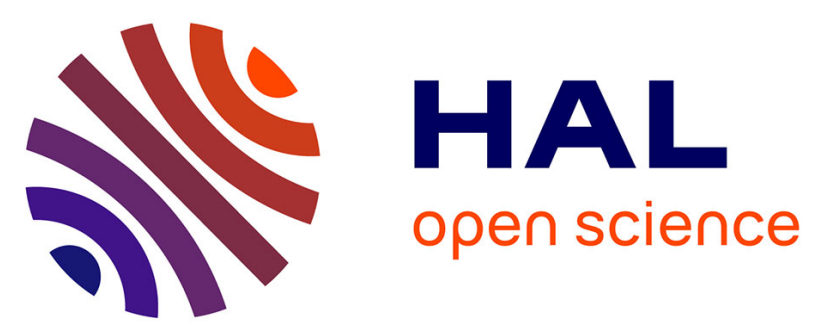

\title{
Mechanism of aggregate formation in simplified industrial silica styrene-butadiene nanocomposites: effect of chain mass and grafting on rheology and structure
}

Guilhem Baeza, Anne-Caroline Genix, Christophe Degrandcourt, Jérémie Gummel, Marc Couty, Julian Oberdisse

\section{To cite this version:}

Guilhem Baeza, Anne-Caroline Genix, Christophe Degrandcourt, Jérémie Gummel, Marc Couty, et al.. Mechanism of aggregate formation in simplified industrial silica styrene-butadiene nanocomposites: effect of chain mass and grafting on rheology and structure. Soft Matter, 2014, 10, pp.6686-6695. 10.1039/c4sm01095g . hal-01110034

\author{
HAL Id: hal-01110034 \\ https://hal.science/hal-01110034
}

Submitted on 27 Jan 2015

HAL is a multi-disciplinary open access archive for the deposit and dissemination of scientific research documents, whether they are published or not. The documents may come from teaching and research institutions in France or abroad, or from public or private research centers.
L'archive ouverte pluridisciplinaire HAL, est destinée au dépôt et à la diffusion de documents scientifiques de niveau recherche, publiés ou non, émanant des établissements d'enseignement et de recherche français ou étrangers, des laboratoires publics ou privés. 


\title{
Mechanism of aggregate formation in simplified industrial silica styrene-butadiene nanocomposites: effect of chain mass and grafting on rheology and structure
}

Guilhem P. Baeza ${ }^{1,2,3}$, Anne-Caroline Genix ${ }^{1,2}$, Christophe Degrandcourt ${ }^{3}$, Jérémie Gummel $^{4}$, Marc Couty $^{3}$, Julian Oberdisse ${ }^{1,2}$

${ }^{1}$ Université Montpellier 2, Laboratoire Charles Coulomb UMR 5221, F-34 095, Montpellier, France

${ }^{2}$ CNRS, Laboratoire Charles Coulomb UMR 5221, F-34095, Montpellier, France

${ }^{3}$ Manufacture Française des Pneumatiques MICHELIN, Site de Ladoux, 23 place des

Carmes Déchaux, F-63 040 Clermont-Ferrand, Cedex 9, France

${ }^{4}$ European Synchrotron Radiation Facility, ESRF, 6 rue Jules Horowitz, BP 220, F38 043, Grenoble, Cedex 9, France

$10 / 07 / 14$

\begin{abstract}
:
The formation of aggregates in simplified industrial styrene-butadiene nanocomposites with silica filler has been studied using a recent model based on a combination of electron microscopy, computer simulations, and small-angle X-ray scattering. The influence of the chain mass (40 to $280 \mathrm{~kg} / \mathrm{mol}$, PI $<1.1$ ), which sets the linear rheology of the samples, was investigated for a low $(9.5 \% \mathrm{v})$ and high $(19 \% \mathrm{v})$ silica volume fraction. $50 \%$ of the chains bear a single graftable end-group, and it is shown that the (chain-mass dependent) grafting density is the structure-determining parameter. A model unifying all available data on this system is proposed and used to determine a critical aggregate grafting density. The latter is found to be closely related to the mushroom to brush transition of the grafted layer. To our best knowledge, this is the first comprehensive evidence for the control of the complex nanoparticle aggregate structure in nanocomposites of industrial relevance by the physical parameters of the grafted layer.
\end{abstract}

\section{Tables: 2}

Figures: 8 


\section{Introduction}

Nanocomposites made of hard inorganic nanoparticles (NPs) embedded in a soft polymer matrix may have mechanical, electrical, or thermal properties of interest for various applications ${ }^{1-4}$. These properties depend to a high extent on the characteristics of the dispersion of the filler NPs in the matrix, which may be individual, or in the form of aggregates, or networks ${ }^{5-10}$. Different types of dispersion may be achieved with different starting materials: in model systems, well-defined colloidal particles allow for a better structural control 6, 11 than pre-aggregated and polydisperse NP-powders used in industrial applications ${ }^{10,12,13}$. Within a given filler system, the structure can be tuned, by varying either the process conditions, which is particular relevant for industrial applications, or the colloidal interactions between NPs. The latter depend strongly on the surface properties of the NPs, where grafting of small (coating) or large (polymer brushes) molecules are methods of choice. These interactions also influence process conditions, like the viscosity during mixing, which is easily modified by changing the mass of the polymer chains ${ }^{14-16}$.

The dispersion of the NPs in model systems has been found to depend on both grafting density and grafted chain mass, via the following mechanisms: (a) For too low grafting densities (typically below the establishment of a brush), NPs are not stabilized and tend to aggregate in a so-called allophobic regime ${ }^{14,17,18}$. (b) As soon as brushes are formed, NPs grafted with long chains (i.e., longer than the matrix chains) may increase the compatibility with the matrix due to wetting of the brush by the shorter matrix chains. This leads to swollen brushes favoring a better state of dispersion 16, 19, 20 . (c) Short brushes usually favor aggregation due to the entropic penalty for the longer matrix chains to wet the brush (autophobic regime ${ }^{14}$ ). (d) In a similar way, very high grafting densities may lead to 'dry' polymer layers, i.e. not wetted by the matrix chains, which causes attractive interactions and aggregation ${ }^{21}$.

In systems with industrial, highly disorder filler, similar mechanisms are expected to affect the final dispersion state, but there is little quantitative information available in the literature, among others due to the intrinsic difficulties to actually characterize the aggregate structure. In a series of papers, we have conducted a systematic study of the dispersion state of simplified industrial nanocomposites formulated by melt mixing of styrene-butadiene (SB) chains with silica pellets of industrial origin ${ }^{22-24}$. A fraction \%D3 of chains may bear a single grafting function, and all nanocomposites are made with both 
matrix and grafted chains of the same mass. The influence of three parameters has been investigated: silica volume fraction, matrix composition in terms of the fraction of graftable chains, and chain mass. The dispersion of the silica NPs was described in a first article in terms of size and mass of aggregates ${ }^{22}$. The latter have been characterized with an original model based on a quantitative combination of transmission electron microscopy (TEM) with synchrotron small-angle X-ray scattering (SAXS). The finding was that the silica NPs are organized in small aggregates containing some forty beads, surprisingly independent of silica volume fraction and thus mixing viscosity. In a second article, we have studied the effect of fraction of graftable matrix chains on the aggregate size $^{23}$. There, it has been shown that the mass of the aggregates can be decreased by about a factor of four using grafting. In a letter, finally, "twin samples", i.e. nanocomposites with identical grafting density but different chain mass were shown to have identical structures ${ }^{24}$.

In this article, a systematic investigation of the influence of the chain mass on the nanocomposite properties and in particular aggregate structure is presented. Different chain masses (40 to $280 \mathrm{~kg} / \mathrm{mol}$ ) induce different mixing viscosities, but also different grafting densities because each graftable chain has a single grafting end-function. Longer chains thus lead to lower grafting densities. The aim of the study is to draw conclusions on the mechanisms of aggregate formation in the mixing process from the evolution of aggregate structure. After the materials and methods section (2), the results are presented in section 3. Starting with the mixing of the nanocomposites (3.1) as a function of chain length, the rheology of the pure and filled matrices (3.2) is discussed. This characterization highlights the strong changes in relaxation time when the chain mass is varied. In section 3.3, the structure of the nanocomposites is studied by SAXS. The data are analyzed in terms of aggregate size from Kratky plots, as well as average aggregation numbers using our coupled SAXS-TEM model. It is based on a measurement by TEM of the volume fraction of (possibly fractal) branches containing aggregates, $\Phi_{\text {fract }}$, reported in the SI. In the discussion, a unified model of the aggregate size dependence on all experimental parameters (chain mass, matrix composition, silica volume fraction) is proposed and compared to all available data. 


\section{Materials and methods}

Nanocomposite formulation: Simplified industrial nanocomposites with silica and SB chains of different mass have been formulated in an internal mixer. The final temperature was set to $160 \pm 5^{\circ} \mathrm{C}$. The size distribution of the NPs constituting the silica pellets (Zeosil 1165 MP from Solvay) obeys a log normal law in agreement with TEM studies $\left(\mathrm{R}_{0}=\right.$ $8.55 \mathrm{~nm}, \sigma=27 \%$, leading to the average bead volume of $\mathrm{V}_{\mathrm{si}}=3.610^{3} \mathrm{~nm}^{3}$ ). This silica has the nominal specific surface of $160 \mathrm{~m}^{2} / \mathrm{g}$. The silica volume fraction in the nanocomposites $\Phi_{\mathrm{si}}$ has been determined by thermogravimetric analysis (Mettler Toledo). The styrene-butadiene random copolymer chains have been purpose-synthesized by Michelin. SB chains were generated by anionic polymerization ${ }^{23}$ and their masses are $\mathrm{M}_{\mathrm{SB}}=40,80,140$, and $280 \mathrm{~kg} / \mathrm{mol}$, all with polydispersity index (PI) below 1.1 as measured by size exclusion chromatography. By measuring the mass distribution of extracted chains, it has been checked that the PI is only very slightly changed by the nanocomposite mixing. The fraction of graftable chains bearing a single graftable endfunction is called the matrix composition and denoted $\% \mathrm{D} 3$, where D3 refers to the silanol one-end functionalized chain $\mathrm{SB}-\mathrm{SiMe}_{2}-\mathrm{OH}$. We define the nominal grafting density of the polymer chains on the available silica surface as:

$$
\rho_{\mathrm{D} 3}=\frac{\left(1-\Phi_{\mathrm{si}}\right) \mathrm{N}_{\mathrm{A}} \% \mathrm{D} 3 \mathrm{~d}_{\mathrm{SB}} \mathrm{R}_{0} \exp \left(2.5 \sigma^{2}\right)}{3 \mathrm{M}_{\mathrm{SB}} \Phi_{\mathrm{si}}}
$$

where $\mathrm{d}_{\mathrm{SB}}=0.94$ g.cm ${ }^{-3}$ is the density of the polymer, and $\mathrm{N}_{\mathrm{A}}$ is the Avogadro number. Further details may be found in references ${ }^{22,23}$. In Table 1, the characteristics of nanocomposites formulated at $50 \% \mathrm{D} 3$ with the different chains at low $(\approx 9.5 \% \mathrm{v})$ and high $(\approx 19 \%$ v) silica volume fraction are summarized.

\begin{tabular}{|c|c|c|c|c|}
\hline $\begin{array}{l}M_{\mathrm{SB}} \\
(\mathrm{kg} / \mathrm{mol})\end{array}$ & $\begin{array}{l}\Phi_{\text {si }}(\text { TGA, } \\
\text { average } 9.5 \% \mathrm{v})\end{array}$ & $\begin{array}{l}\rho_{\mathrm{D} 3} \\
\left(10^{-3} \mathrm{~nm}^{-2}\right)\end{array}$ & $\begin{array}{l}\Phi_{\mathrm{si}}(\text { TGA, } \\
\text { average } 19 \% \mathrm{v})\end{array}$ & $\begin{array}{l}\rho_{\mathrm{D} 3} \\
\left(10^{-3} \mathrm{~nm}^{-2}\right)\end{array}$ \\
\hline 40 & $10.4 \% \mathrm{v}$ & 208 & $20.1 \% \mathrm{v}$ & 96 \\
\hline 80 & $9.8 \% \mathrm{v}$ & 111 & $19.5 \% \mathrm{v}$ & 50 \\
\hline 140 & $8.4 \% \mathrm{v}$ & 75 & $16.8 \% \mathrm{v}$ & 34 \\
\hline 280 & $9.5 \% \mathrm{v}$ & 33 & $19.4 \% \mathrm{v}$ & $\overline{14}$ \\
\hline
\end{tabular}

Table 1: Nanocomposite samples formulated with chains of different mass at fixed matrix composition (50\%D3). 
System characterization: SAXS experiments were performed on beamline ID2 $(1.1 \AA$, $12.46 \mathrm{keV}, 0.001$ to $\left.0.5 \AA^{-1}\right)$ and on its Bonse-Hart set-up $\left(\mathrm{q}_{\min }=10^{-4} \AA^{-1}\right)$ at ESRF (Grenoble). Sample thickness was $\approx 0.8 \mathrm{~mm}$. Our scattered intensity $\mathrm{I}(\mathrm{q})$ denotes the scattering cross section per unit sample volume $\mathrm{d} \Sigma / \mathrm{d} \Omega\left(\right.$ in $\mathrm{cm}^{-1}$ ). It was obtained with standard procedures given by ESRF. The contrast of silica in polymer in SAXS experiments can be deduced from the scattering length densities $\left(\rho_{\mathrm{SB}}=8.8510^{10} \mathrm{~cm}^{-2}\right.$, $\left.\rho_{\mathrm{SiO} 2}=1.9710^{11} \mathrm{~cm}^{-2}, \Delta \rho=1.0910^{11} \mathrm{~cm}^{-2}\right)$.

Linear rheology: A stress-controlled rheometer AR 2000 in the strain-controlled mode was used to study the rheological response in the linear regime of the nanocomposites (plate-plate geometry, $20 \mathrm{~mm}$ diameter) with frequency sweeps at fixed low deformation level $(\gamma=0.1 \%)$ between $10^{\circ} \mathrm{C}$ and $80^{\circ} \mathrm{C}$. Using time-temperature superposition, master curves at $50^{\circ} \mathrm{C}$ of the storage $\mathrm{G}^{\prime}(\omega)$ and loss $\mathrm{G}^{\prime \prime}(\omega)$ moduli were constructed between $\omega=2 \pi \mathrm{f}=2 \pi 10^{-3}$ and $2 \pi 10^{3} \mathrm{rad} / \mathrm{s}$. In addition, dynamic mechanical analysis (DMA) has been performed on a Rheometrics RDAII instrument with the same control strain $(0.1 \%)$. The sample geometry was rectangular with average dimensions of $12 \times 7.5 \times 1.5 \mathrm{~mm}^{3}$. Temperature sweeps at $10 \mathrm{~Hz}$ were measured from $-80^{\circ} \mathrm{C}$ to $80^{\circ} \mathrm{C}$ (temperature step $3 \mathrm{~K}$, soak time of $60 \mathrm{~s}$ ).

Structural analysis: The SAXS intensities are analyzed quantitatively using input from the TEM analysis, a model for a polydisperse hard sphere structure factor, and a polydisperse form factor, as explained in detail in ref. ${ }^{22}$. The TEM observations allow the determination of the fraction of branches containing aggregates. Results as a function of matrix composition at fixed mass have been published previously ${ }^{23}$. The TEM pictures used for the present article are shown in the SI. They show that the fraction of branches for different polymer masses are fully compatible with the previously published data. Size information is taken from the observation of the position $\mathrm{q}_{\mathrm{agg}}$ of breaks in slope of $\mathrm{I}(\mathrm{q})$, which can be highlighted with a Kratky plot, $\mathrm{q}^{2} \mathrm{I}(\mathrm{q})$ vs $\mathrm{q}$. The outcome is the average radius of aggregates, $R_{\mathrm{agg}}=\pi / \mathrm{q}_{\mathrm{agg}}$, as well as the number of silica NPs making up the aggregate, $\mathrm{N}_{\mathrm{agg}}$. The latter is obtained by dividing the total silica volume of an aggregate by the average volume of a single $\mathrm{NP}, \mathrm{V}_{\mathrm{si}}$ :

$$
\mathrm{N}_{\mathrm{agg}}=\frac{\mathrm{V}_{\text {si in agg }}}{\mathrm{V}_{\mathrm{si}}}
$$


$\mathrm{N}_{\text {agg }}$ and $\mathrm{R}_{\mathrm{agg}}$ are related by the aggregate compacity $\kappa$, which we define as the internal silica volume fraction of the aggregates:

$$
\kappa=\frac{\mathrm{V}_{\text {si in agg }}}{\mathrm{V}_{\mathrm{agg}}}=\frac{\mathrm{N}_{\mathrm{agg}} \mathrm{V}_{\mathrm{si}}}{\frac{4}{3} \pi \mathrm{R}_{\mathrm{agg}}^{3}}
$$

\section{Results}

\subsection{Nanocomposite mixing rheology as a function of chain mass}

A strong effect on the (non linear) mixing rheology is obtained for different chain mass, due to the variation of the terminal relaxation time and thus viscosity with chain length ${ }^{25}$. Here, the chain mass is varied from 40 to $280 \mathrm{~kg} / \mathrm{mol}$, and the torque-level increases considerably. This is shown in Figure 1 , for nanocomposites containing $\approx 9.5 \% \mathrm{v}$ silica (cf. Table 1), with fixed matrix composition (50\%D3). After the addition of the silica, the overall shape of the curve remains relatively flat, even at high viscosity (high mass). At constant mass $(140 \mathrm{~kg} / \mathrm{mol})$, a decrease may be noticed at high volume fractions, presumably related to the even higher viscosity for $\Phi_{\mathrm{si}}>13 \%{ }^{22}$. An inversion in torque between 140 and $280 \mathrm{~kg} / \mathrm{mol}$ is observed, which may be due to wall slip in too viscous samples leading to the measurement of a lower apparent viscosity.

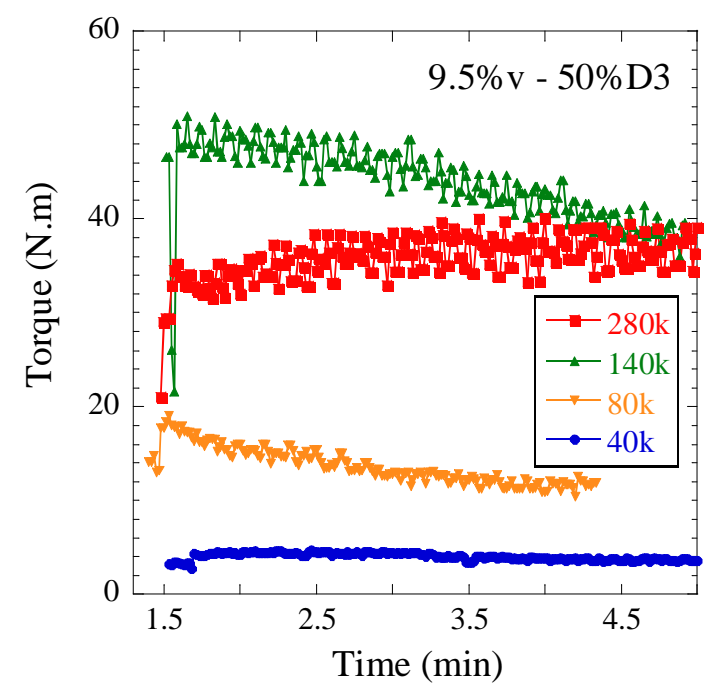

Figure 1: Torque observed during mixing of SB nanocomposites for a series in chain mass $\left(40,80,140\right.$, and $\left.280 \mathrm{~kg} / \mathrm{mol}, \Phi_{\mathrm{si}} \approx 9.5 \% \mathrm{v}, 50 \% \mathrm{D} 3\right)$. 
In a previous article ${ }^{23}$, we have discussed the influence of the fraction of graftable chains on the mixing rheology: the observed torque in the mixer increases weakly with the matrix composition \%D3. Here, the nominal \%D3-value remains constant $(50 \%$ of the chains are graftable), but the number of chain ends per unit silica surface decreases with increasing chain mass. According to eq. (1), the nominal grafting density $\rho_{\mathrm{D} 3}$ given in Table 1 decreases thus by a factor of seven. The torque increase with chain mass shown here thus demonstrates that the chain mass dominates the mixing process, and it outweighs by far any contribution from the grafting. One may also note that the increase in torque - for comparison a factor of ten after 2.5 minutes between the lowest and the highest torque - is less strong than the increase in relaxation time (typically by a factor greater than 500) and viscosity with chain mass, which will be discussed below.

\subsection{Matrix and nanocomposite rheology as a function of chain mass}

The effect of chain mass also dominates the linear rheological response of the silica-free matrices. In Figure $2 a$, the storage modulus $G^{\prime}(\omega)$ is plotted for matrices with $M_{S B}=40$ to $280 \mathrm{~kg} / \mathrm{mol}$. These curves have the typical shape of the moduli of entangled polymer melts: they converge to a high-frequency rubbery plateau $\mathrm{G}_{0}$ in the range between 0.8 and 1.0 $\mathrm{MPa}$. This plateau is quite flat, which is a characteristic feature of the narrow mass distributions of the polymers used in this study. Next, the moduli possess a chain-length dependent characteristic crossover located at $1 / \tau$ to a low-frequency flow regime. Here $\tau$ is the terminal relaxation time, and in the flow regime $G^{\prime}(\omega)$ is given by $G_{0}(\omega \tau)^{\beta}$. For ideal melts, $\beta$ should be 2, whereas it is between 1.2 for the higher masses and 1.6 for the lower ones here. The corresponding loss moduli with their characteristic peaks are shown in Figure 2b. Again the power law of G" in the flow regime $-G^{\prime \prime}(\omega)=\eta_{0} \omega-$ is not exactly verified, as the exponent decreases from almost one (0.98) to 0.75 with increasing mass. From the peak position, relaxation times $\tau=1 / \omega_{\text {peak }}$ are found to evolve by several orders of magnitude with chain mass. This evolution is compared in the inset of Figure $2 b$ to the usual power law of linear chains. 

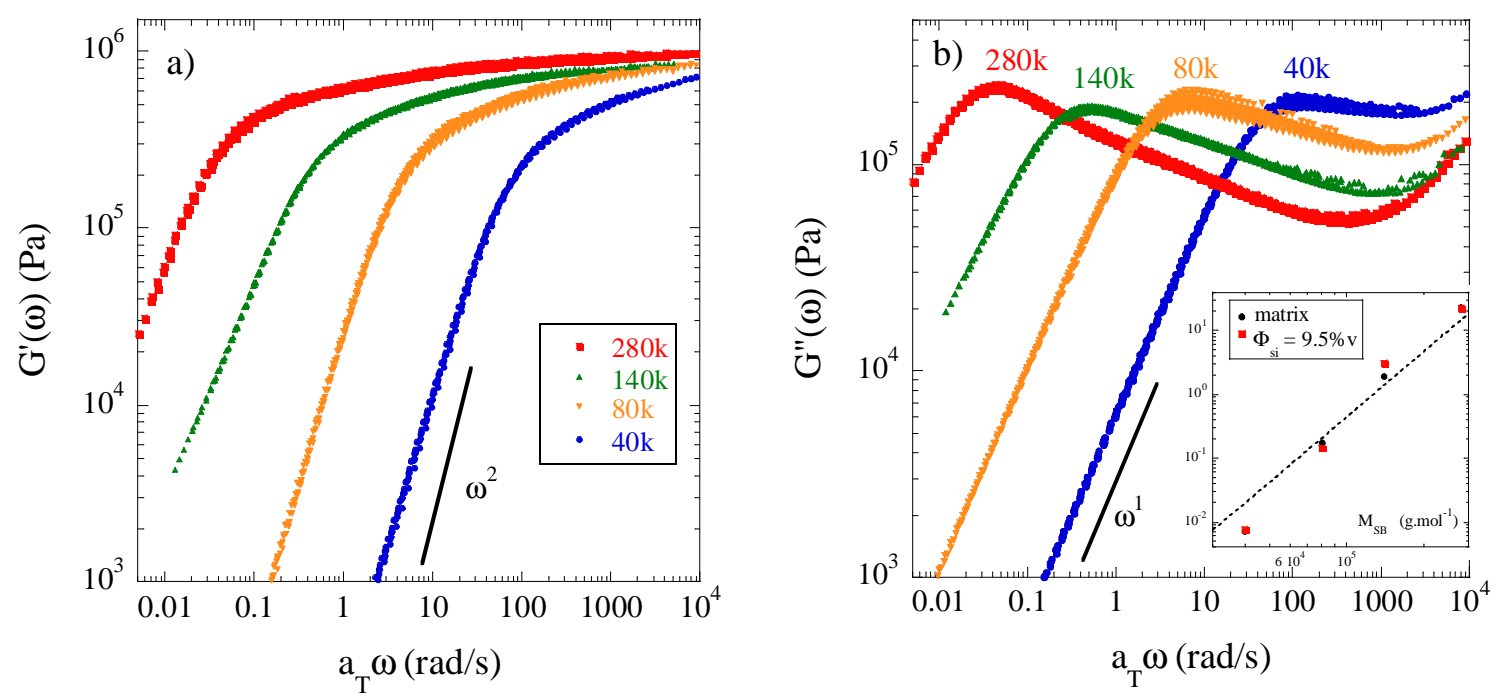

Figure 2: Master curves at the reference temperature of $50^{\circ} \mathrm{C}$ for (a) the storage moduli $\mathrm{G}^{\prime}(\omega)$ for pure polymer matrices of composition $50 \% \mathrm{D} 3$, with different chain mass (40, 80,140 , and $280 \mathrm{~kg} / \mathrm{mol}$ ), and (b) the corresponding loss moduli G' $(\omega)$. The inset shows the evolution of the relaxation time with mass for matrices and $9.5 \% \mathrm{v}$-nanocomposites as compared to a $\mathrm{M}^{3.4}$ power law.

The rheological properties of the samples evolve as silica is added to the matrices. In Figures $3 a$ and $b$, the moduli $G^{\prime}(\omega)$ and $G^{\prime \prime}(\omega)$ are plotted for nanocomposites with ca. $9.5 \% \mathrm{v}$ of silica, for the four different chain masses. The arrow in Figure 3a demonstrates the scattering of the data: more precise measurements of the plateau modulus have been performed by DMA, and the data normalized to the DMA value. The graphs resemble the ones of the pure matrices, in the sense that a flow regime and a plateau can be distinguished in G', and a peak in G'. The effect of the chain mass on the peak position is shown via the relaxation time together with the pure matrix case in the inset of Figure $2 b$. The relaxation time is found to increase with a power-law exponent comparable to the one observed in absence of silica and compatible with 3.4. Within error bars, the relaxation time does not seem to evolve from the matrix to the loaded sample. Consequently, the dynamics of the nanocomposites probed in this frequency range appears to be similar to the pure melt one. 

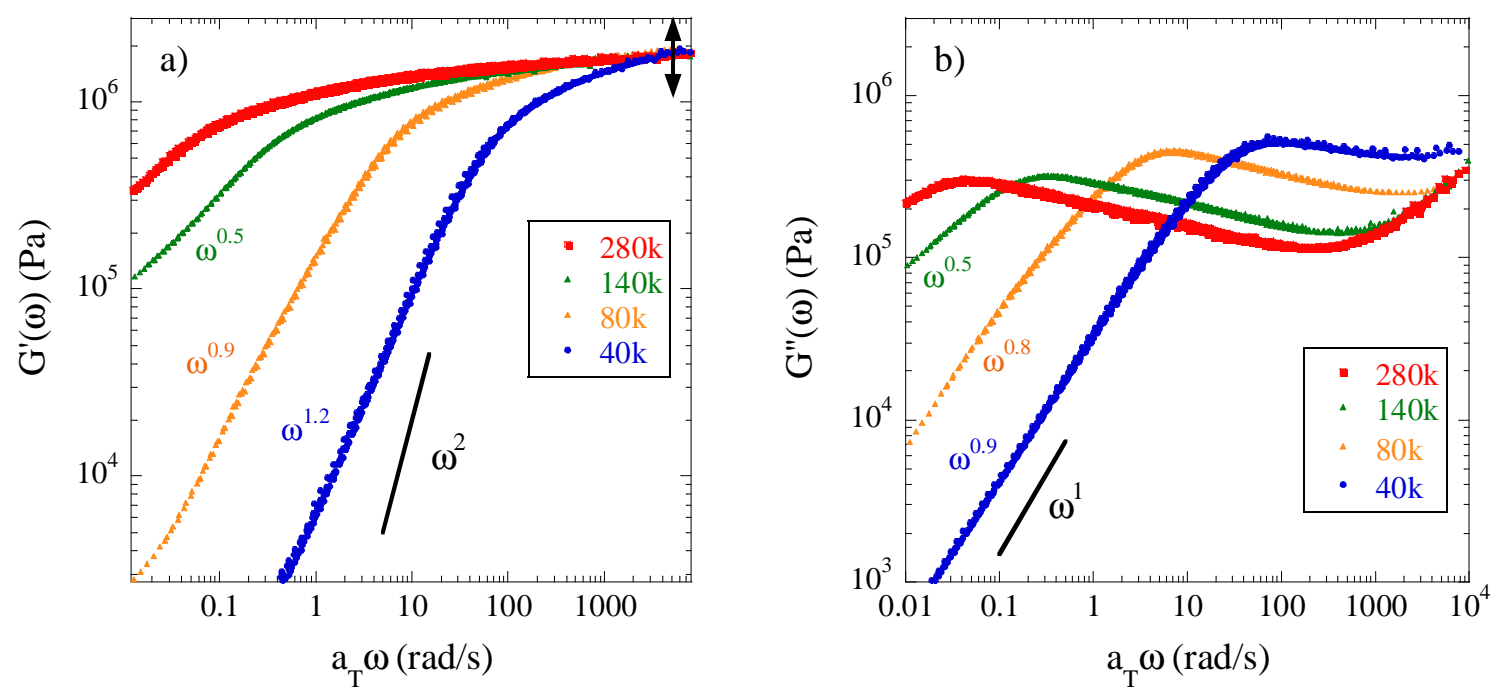

Figure 3: Master curves at the reference temperature of $50^{\circ} \mathrm{C}$ for (a) $\mathrm{G}^{\prime}(\omega)$ and (b) $\mathrm{G}$ ' $(\omega)$ for nanocomposites $(\approx 9.5 \% \mathrm{v} ; 50 \% \mathrm{D} 3 ; 40,80,140$, and $280 \mathrm{~kg} / \mathrm{mol})$. The arrow indicates the scattering of the moduli which have been renormalized to the corresponding DMA values.

The slopes and the plateau moduli of the data shown in Figure $3 \mathrm{a}$ and $\mathrm{b}$, however, are different from the pure-matrix values. The $\mathrm{G}_{0}$ are higher, which is a consequence of the reinforcement effect. The low-frequency slopes of the storage moduli evolve from 1.2, i.e. well below their Maxwellian value of two, to 0.5 (for 40 to $140 \mathrm{~kg} / \mathrm{mol}$, respectively, the highest mass slope being out of the window). The corresponding slopes of the loss moduli decrease as well, from 0.9 at low mass - which is close to the expected exponent of one - to 0.5 . These values suggest that the flow regime is not properly reached, although most of the sample relaxes at $\omega<1 / \tau$. Indeed, identical power law exponents close to $1 / 2$ for both $G^{\prime}$ and $G^{\prime}$ ' have been reported to be a signature of gel-like rheology ${ }^{26}$, ${ }^{27}$. Higher mass nanocomposites thus have non relaxing, elastic contributions to the stress, which is due to a filler network, as typically encountered with nanocomposites ${ }^{23,28,29}$. In the present study, the higher chain mass pushes the system to higher elasticity in the low frequency range, presumably as a different dispersion state of the filler is reached.

\subsection{Filler structure in nanocomposites as a function of chain mass}

As with the rheological data presented in the previous section, our objective here is to characterize the impact of the chain mass on the structure of the silica in the polymer matrix. In Figure 4, the scattered intensities of nanocomposites of fixed matrix 
composition $(50 \% \mathrm{D} 3)$, for two silica volume fractions $(\approx 9.5 \% \mathrm{v}$ and $\approx 19 \% \mathrm{v}$, respectively, see Table 1 for exact values), are shown for the four different chain masses.
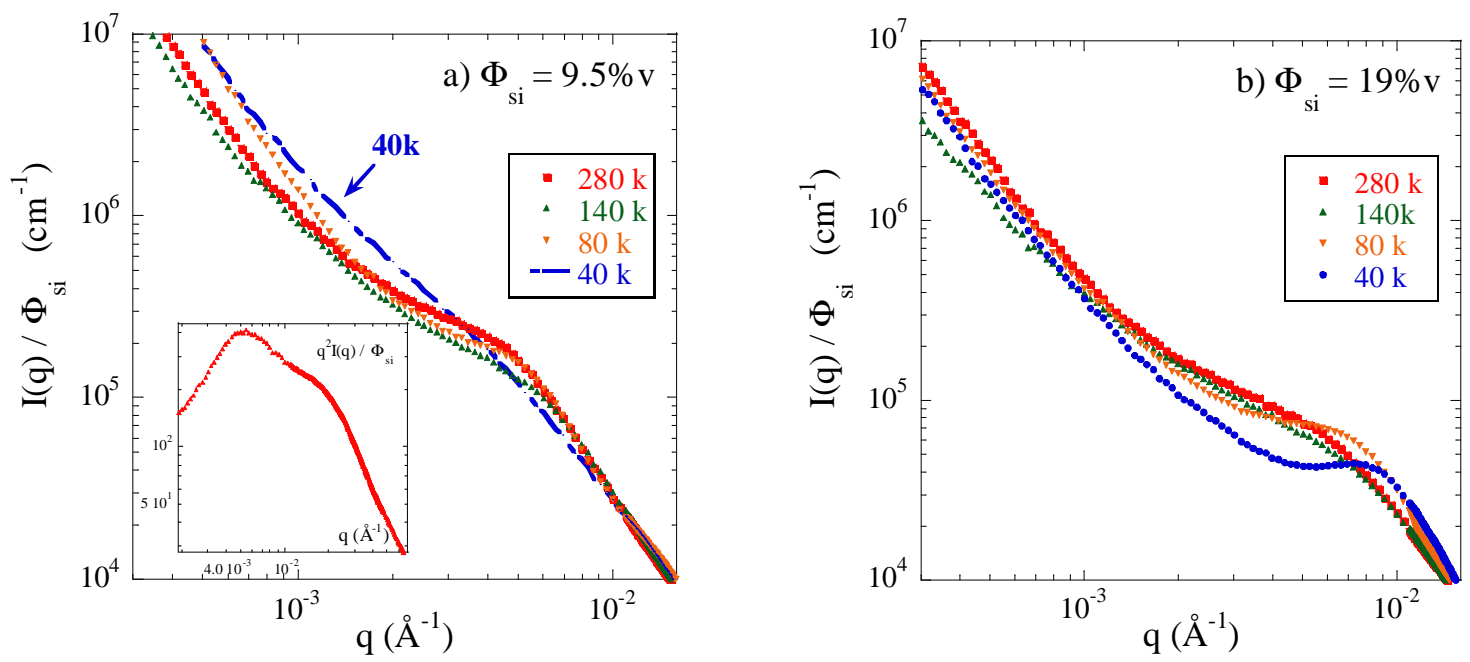

Figure 4: Silica structure in nanocomposites. (a) Reduced SAXS intensity $\mathrm{I}(\mathrm{q}) / \Phi_{\text {si }}$ with $\Phi_{\mathrm{si}} \approx 9.5 \% \mathrm{v}$, D3-fraction $50 \%$, for various matrix chain masses: 40, 80, 140, $280 \mathrm{~kg} / \mathrm{mol}$. In the inset, the Kratky representation of the 280k-data. (b) Same result for $\Phi_{\mathrm{si}} \approx 19 \% \mathrm{v}$.

We start the discussion with the structure of the low concentration nanocomposites in Figure 4a. First, a singular sample needs to be discussed. Unlike all other samples, the $10.4 \% \mathrm{v}$ silica sample with the $40 \mathrm{~kg} / \mathrm{mol}$ chains is a highly viscous liquid, which induces a very low torque in the mixer (Figure 1). Intuitively, one suspects that such a low torque is insufficient to break down the silica pellets into nano-sized objects. We have therefore measured the structure of the silica pellet powder by SAXS, using sticky tape as a sample holder. Due to the imprecise sample mass in the beam, the resulting intensity is not in absolute units, but its shape can be compared to the one of the viscous sample, which is done in Figure 5. 


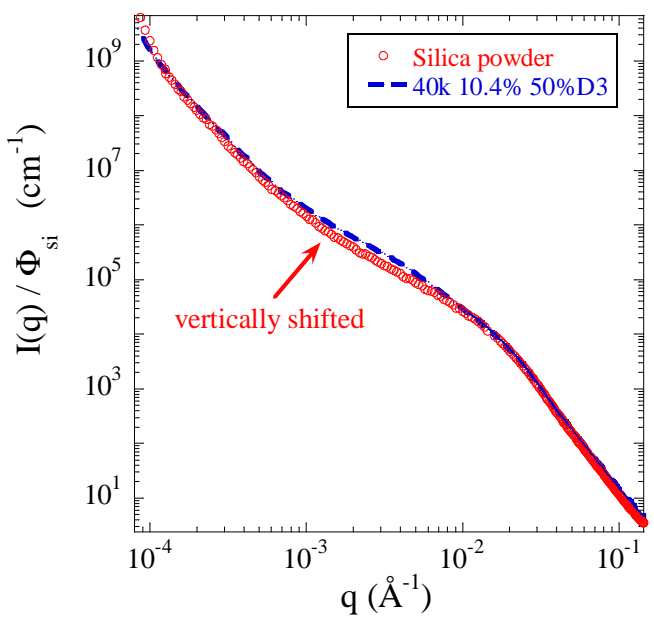

Figure 5: Silica structure in nanocomposite $\left(\Phi_{\mathrm{si}}=10.4 \% \mathrm{v}, 50 \% \mathrm{D} 3,40 \mathrm{~kg} / \mathrm{mol}\right)$ compared to structure of pure filler dispersed on sticky tape and renormalized to the same high-q values.

The superposition of the scattering curves in Figure 5 indicates that the structures of silica on the sub-micron length scale probed in this experiment are close. It can thus be concluded that the viscous nanocomposite sample contains only dispersed original silica powder, without fragmentation on the nanoscale. This explains why the scattered intensity in Figure 4a of this sample is also quite different from the others, which have thus undergone filler fragmentation. This sample is ignored in the following discussion.

The remaining scattering curves in Figure 4a display the same features as all curves discussed before ${ }^{22,23}$ : (a) the low-q upturn which is the signal of large-scale structures, (b) the breaks in slope and plateau-like features in the intermediate q-range which reveal a typical aggregate size and mass, and (c) the large angle scattering reminiscent of the silica NP structure. We now apply a recently developed model to the structural analysis of these samples ${ }^{22}$, starting with the subtraction of the low-q power law. The breaks in slope can be highlighted using a Kratky plot $-q^{2} I(q)$ vs. $q-$ as exemplified in the inset of Figure $4 \mathrm{a}$. There the two peaks due to aggregate and silica bead interactions (close contact) are easily identified. Fitting a double log-normal to the peaks ${ }^{22,23}$, the lognormal parameter identifying the low-q peak at $\mathrm{q}_{\mathrm{agg}}$ can be determined. This procedure gives the average aggregate radius $R_{\mathrm{agg}}=\pi / \mathrm{q}_{\mathrm{agg}}$, and we find $\mathrm{R}_{\mathrm{agg}}=45.4,41.3$, and 45.9 $\mathrm{nm}$, for 80,140 , and $280 \mathrm{~kg} / \mathrm{mol}$, with an errorbar of $\pm 2 \mathrm{~nm}$. It appears that these radii do not follow the order of the masses. Using the volume fraction of fractal branches as determined by TEM (see SI), the average compacity of aggregates can be extracted from 
the data in Figure 4a. It varies between 30 and 40\%, and allows the estimation of the average aggregation number. The latter follows the evolution of $R_{a g g}$, i.e. $N_{a g g}$ decreases from about 100 to 50 and then goes up to 100 again. The outcome of this structural modeling is summarized in Table 2.

\begin{tabular}{|r|r|r|r|l|r|r|r|r|}
\hline \multirow{2}{*}{\begin{tabular}{l}
$\mathbf{M}_{\text {SB }}(\mathbf{k g} / \mathbf{m o l})$ \\
\cline { 2 - 10 }
\end{tabular}} & \multicolumn{4}{|c|}{$\Phi_{\text {si }}=9.5 \% \mathbf{v}$} & \multicolumn{4}{|c|}{$\Phi_{\text {si }}=19 \% \mathbf{~}$} \\
\hline 40 & - & - & - & 31.3 & $77 \%$ & $36 \%$ & 25 & 29.7 \\
\hline 80 & $54 \%$ & $36 \%$ & 86 & 45.4 & $75 \%$ & $38.5 \%$ & 39 & 33.8 \\
\hline 140 & $59 \%$ & $30.5 \%$ & 51 & 41.3 & $73 \%$ & $34.5 \%$ & 44 & 36.8 \\
\hline 280 & $47.5 \%$ & $40.5 \%$ & 99 & 45.9 & $74.5 \%$ & $39 \%$ & 65 & 40.1 \\
\hline
\end{tabular}

Table 2: Structural parameters of nanocomposite samples for different chain mass, two silica volume fractions, and at fixed matrix composition (50\%D3).

The series in chain mass at $\approx 19 \% \mathrm{v}$ silica volume fraction shown in Figure $4 \mathrm{~b}$ is treated following the same procedure. The low-q power laws are subtracted, and the intensity level is used to calculate the average aggregate compacity. The aggregate radii are determined by the Kratky plots, giving $\mathrm{R}_{\mathrm{agg}}=29.7$, 33.8, 36.8, and $40.1 \mathrm{~nm}$, for 40 to 280 $\mathrm{kg} / \mathrm{mol}$, respectively. Combining the compacity with the radius gives again $\mathrm{N}_{\mathrm{agg}}$, and these numbers are also given in Table 2. It is remarkable that the intensity curves are rather close in Figure $4 \mathrm{a}$ and $\mathrm{b}$, but do not follow the order of the chain mass. In the case of the low silica volume fraction $(\approx 9.5 \% \mathrm{v})$, the model reproduces this non monotoneous feature. At high silica volume fraction $(\approx 19 \% \mathrm{v})$, it extracts a monotonously increasing aggregation number with chain mass. This is because the model uses two key characteristics of the intensity curves, the position of the break in slope, and the low-q intensity, which evolve differently with mass. Finally, $\mathrm{N}_{\text {agg }}$ more than doubles from 40 to $280 \mathrm{~kg} / \mathrm{mol}$ at $19 \% \mathrm{v}$, while the compacity stays again approximately constant in the 35 $40 \%$ range. 


\section{Discussion}

It is not easy to identify a general tendency of the structure dependence on chain mass with the data given in Table 2. In the intermediate-q domain of interest for aggregate structure $\left(\approx 0.003 \AA^{-1}\right)$, the intensities shown in Figure 4 are in the same range. Our analysis shows that this is due to both relatively close $R_{\text {agg }}$ values and close compacities, with weak and - for the lower volume fraction - non monotonic evolutions. The compacity staying in the $30-40 \%$ range, one can discuss either $\mathrm{R}_{\mathrm{agg}}$ or $\mathrm{N}_{\mathrm{agg}}$. In Figure 6, the average aggregate radius $\mathrm{R}_{\mathrm{agg}}$ is plotted as a function of chain mass for these samples.

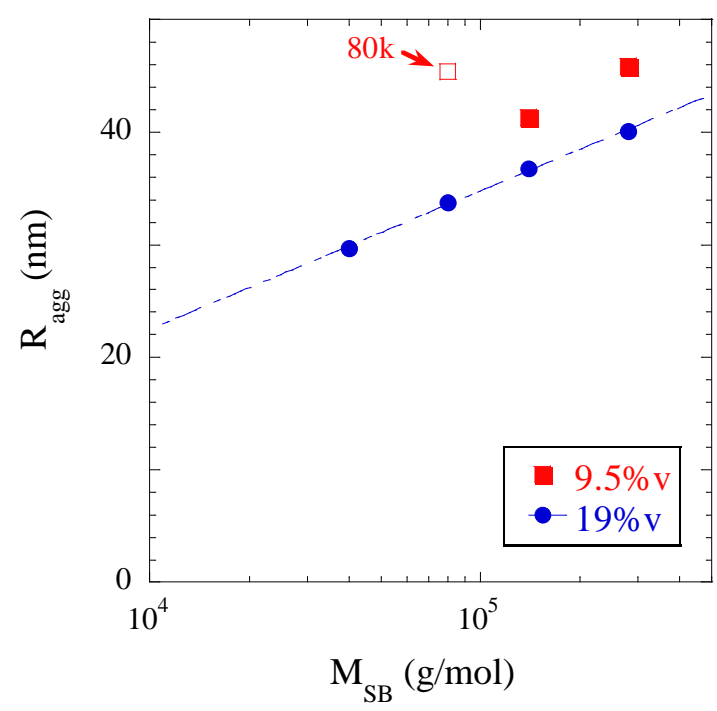

Figure 6: Average radius in $\mathrm{nm}$ of silica aggregates in nanocomposite for low $(\approx 9.5 \% \mathrm{v})$ and high $(\approx 19 \% \mathrm{v}$ ) silica volume fractions (matrix composition 50\%D3). The broken line is a guide to the eye. The $9.5 \% \mathrm{v}-80 \mathrm{k}$ sample is partially fragmented.

Under the mentioned restrictions, the trend appears to be an increase of aggregate size with chain mass for $19 \% \mathrm{v}$ silica. This is consistent with the decrease of the number of graftable functions, which was previously shown to have the same effect at fixed chain mass ${ }^{23}$. The solid line in the Figure is a logarithmic function $(A+B \ln M)$ used as guide to the eye. A model leading to a linear fitting function will be presented below and compared to all available data.

For the 9.5\%-samples, the relationship is non-monotonic, and a combination with an additional effect must be invoked to explain the data. In Figure 5, the $40 \mathrm{~kg} / \mathrm{mol}$ sample was shown not to fragment due to the too low matrix viscosity. One may argue that these 
aggregates are thus the biggest, whereas they are more fragmented in matrices of longer chains. This leads to a mechanism of decrease of aggregate size with chain mass up to $140 \mathrm{~kg} / \mathrm{mol}$, with presumably partial fragmentation at $80 \mathrm{~kg} / \mathrm{mol}$. At $19 \% \mathrm{v}$, the high amount of silica ensures a high viscosity, and only the effect of the number of grafting ends influences the aggregate size.

We come to the conclusion that once the samples are viscous enough for effective mixing and fragmentation to take place (i.e., all samples but the one shown in Figure 5), it is the density of grafting ends per unit silica surface $\rho_{\mathrm{D} 3}$, as defined in eq. (1), which determines the structure. This hypothesis has been tested successfully in ref. ${ }^{24}$, where the existence of so-called "twins", i.e. samples of identical grafting density having also identical structure, was evidenced. We now generalize this concept and examine the evolution of structure with $\rho_{\mathrm{D} 3}$.

Our goal is to compare all structures of nanocomposites formulated for different silica volume fraction $\Phi_{\mathrm{si}}$, matrix composition $\% \mathrm{D} 3$, and chain mass. Instead of comparing the full intensity curves, we compare the aggregate radii $\mathrm{R}_{\mathrm{agg}}$ obtained with the Kratky plots, and alternatively the average aggregation numbers $\mathrm{N}_{\text {agg }}$. All data are taken from Table 2, Figure 6, and previously published data for the $\Phi_{\mathrm{si}}$ of interest here. In Figure $7 \mathrm{a}$, all $\mathrm{R}_{\mathrm{agg}}{ }^{-}$ values at our disposal are plotted as a function of $\rho_{\mathrm{D} 3}$. These data have been regrouped in two subsets, for $\approx 9.5 \% \mathrm{v}$ and $\approx 19 \% \mathrm{v}$ silica contents. The same is done for $\mathrm{N}_{\text {agg }}$ in Figure $7 b$. 

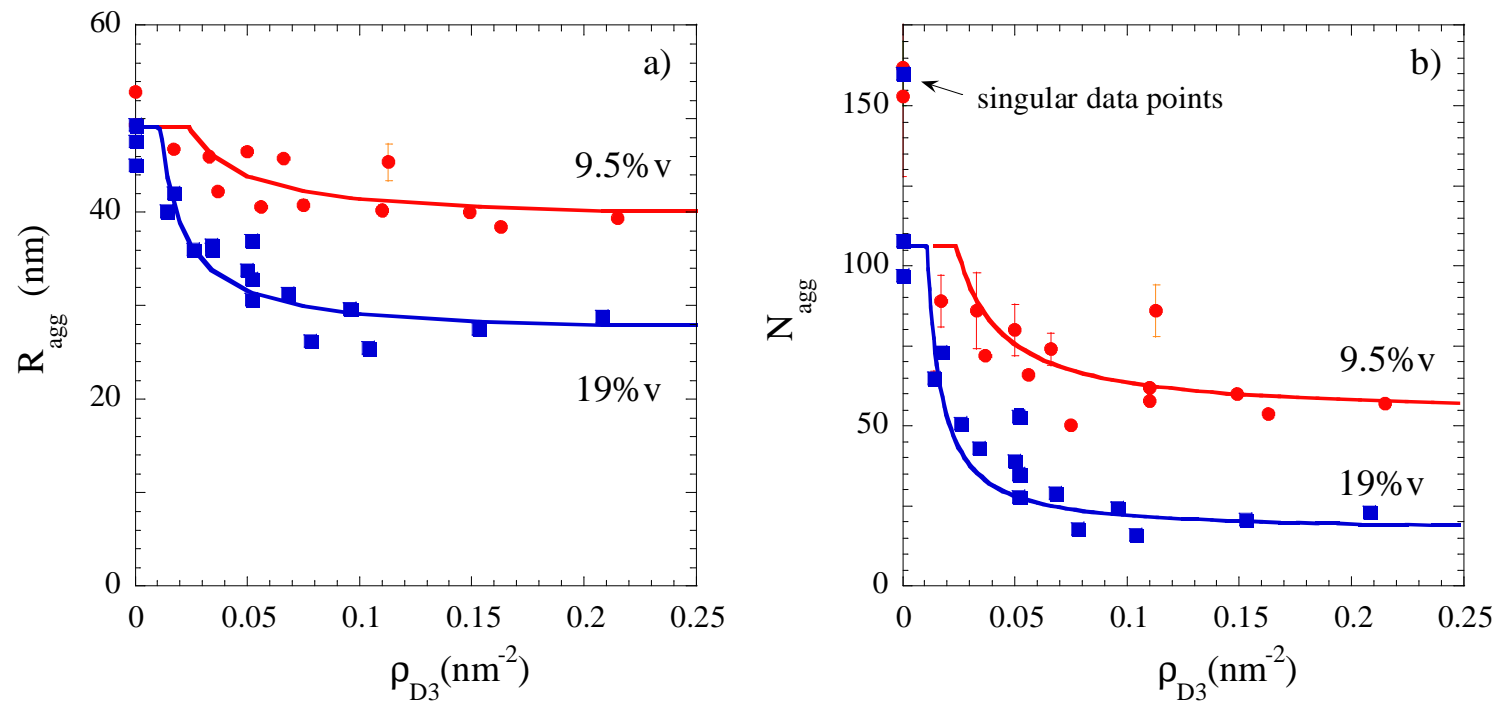

Figure 7: a) Average aggregate radius $\mathrm{R}_{\mathrm{agg}}$ as a function of the nominal grafting density $\rho_{\mathrm{D} 3}$, for all data regrouped in subsets at ca. $9.5 \% \mathrm{v}$ and $19 \% \mathrm{v}$ silica. The fit functions use $\rho_{\mathrm{D} 3}{ }^{\mathrm{c}}=0.01 \mathrm{~nm}^{-2}$ and $\mathrm{R}_{\max }=49.15 \mathrm{~nm}$ for both volume fractions, and $\mathrm{R}_{\min }=39$ and 26.7 $\mathrm{nm}$ for $9.5 \% \mathrm{v}$ and $19 \% \mathrm{v}$ data, respectively, see text for details. b) Average aggregation number $\mathrm{N}_{\mathrm{agg}}$ for the same subsets compared to the model prediction with compacity $\kappa=$ $35 \%$. The points at $\rho_{\mathrm{D} 3}=0$ are singular due to a considerably higher $\kappa$ and not captured by the model with $\kappa$ fixed to $35 \%$.

The data points in Figure 7 are rather scattered for all data sets, but a general tendency is recognized. Error bars are due to scattering in the fraction of branches determined by TEM (cf. SI), and are found to be smaller than the symbols apart from those visible in the graph. Both $\mathrm{R}_{\mathrm{agg}}$ function in Figure 7a start from around $50 \mathrm{~nm}$, then decrease measurably - by some $25 \%$ resp. $40 \%$ of their initial value - , and level off for higher grafting densities. Such a decrease followed by saturation has already been observed in the study of the grafting fraction (\%D3) ${ }^{23}$. In the present article, more data sets are simultaneously described, including the variation of the chain mass and matrix composition \%D3. Both $\mathrm{R}_{\mathrm{agg}}$-sets have been compared to a simple function as derived below:

$$
\mathrm{R}_{\mathrm{agg}}\left(\rho_{\mathrm{D} 3}\right)=\left\{\begin{array}{c}
\mathrm{R}_{\mathrm{max}} \text { for } \rho_{\mathrm{D} 3}<\rho_{D 3}^{m} \\
\mathrm{R}_{\mathrm{min}}+\frac{\mathrm{R}_{\mathrm{Si}}}{\kappa \frac{\rho_{\mathrm{D} 3}}{\rho_{D 3}^{c}}} \text { elsewhere }
\end{array}\right.
$$

Here $\mathrm{R}_{\mathrm{si}}$ is a radius representative of the silica bead radius, and the values of $\mathrm{R}_{\max }$ and $\rho_{D 3}^{m}$ will be discussed below. We use $\mathrm{R}_{\mathrm{si}}=\mathrm{R}_{0}$ and $\kappa=35 \%$ (see Table 2) for simplicity. The 
function in eq.(4) defines a critical grafting density $\rho_{D 3}^{c}$. We find $\rho_{D 3}^{c}=0.01 \mathrm{~nm}^{-2}$ for both. It is obvious from Figure $7 \mathrm{a}$ that $\rho_{\mathrm{D} 3}$ is the structure determining parameter for a given silica volume fraction of such simplified industrial nanocomposites. The critical aggregate grafting density $\rho_{D 3}^{c}$ gives the limit above which the size of the aggregates is not affected any more by the grafting. At high grafting, the aggregate radius saturates at a minimum value $R_{\min }$, which depends on the silica volume fraction. The higher $\Phi_{\text {si }}$, the lower the final aggregate radius $R_{\min }$, i.e., the stronger the breakdown mechanism in the mechanical mixer. In absence of or for low grafting $\rho_{\mathrm{D} 3}<\rho_{D 3}^{m}$ in Figure $7 \mathrm{a}$, the system is not prone to deagglomeration, and a maximum aggregate radius $R_{\max }$ is conserved from the initial silica pellets, independent of silica concentration and thus mixing rheology. In the framework of eq.(4), this is accounted for by introducing a cut-off value $\rho_{D 3}^{m}$ in grafting density, below which the aggregate radius has been set to $R_{\max }=49.15 \mathrm{~nm}$ in the model, for both $\Phi_{\mathrm{si}}$. It is interesting to note that the $10.5 \% \mathrm{v}-40 \mathrm{k}$ sample, which we have shown in Figure 5 to have a nanostructure very similar to the unperturbed powder, has a $\mathrm{R}_{\mathrm{agg}}$ value compatible with $\mathrm{R}_{\max }$ (data and fit shown in $\mathrm{SI}$ ). To summarize, primarily high viscosities seem to be necessary - i.e. either high chain masses or high silica volume fractions - in order to have an efficient break-down; secondly, it is mandatory to have a high grafting density to impede re-agglomeration.

In Figure $7 \mathrm{~b}$, all available data points for $\mathrm{N}_{\mathrm{agg}}$ have been combined in a single plot. Note that $\mathrm{N}_{\mathrm{agg}}$ is the outcome of a calculation which takes polydispersity into account, i.e. it cannot be simply determined using eq.(3) with the average radius for $R_{a g g}$, but eq.(3) has to be applied to each population of the distribution, and averaged. The result displays some singular data points at $\rho_{\mathrm{D} 3}=0$, which correspond to substantially higher compacities, well above $50 \%$. Such values have been used in a separate calculation and confirm the high aggregation numbers. For all other data points in Figure $7 \mathrm{~b}$, we have used the average radius $R_{\text {agg }}$ given in eq.(4) using identical parameters $\left(R_{\min }, R_{\max }, \kappa\right.$, $\rho_{D 3}^{c}$ ), and combined it with the polydispersity and eq.(3). The typical aggregation number as a function of $\rho_{\mathrm{D} 3}$ can then be predicted. The result is shown in Figure $7 \mathrm{~b}$, with again reasonable agreement with the data.

To rationalize these ideas, we have constructed a simple model leading to eq.(4). The nominal grafting density gives the number of grafts per unit surface on each silica NP. In 
Figure $8 \mathrm{a}$, this is illustrated by the ideal - experimentally unreached - dispersion of individual NPs. If NPs are agglomerated, one may suppose that grafting will preferentially take place at the aggregate surface, as illustrated in the next step in Figure 8a. This defines a new aggregate grafting density, which depends on the aggregate size and structure, and which can be expected to be higher than the nominal NP grafting density due to the lower surface. To fix ideas, in small aggregates, containing typically $\mathrm{N}_{\mathrm{agg}}=50 \mathrm{NPs}$, with $\mathrm{R}_{\mathrm{agg}}=50 \mathrm{~nm}$ (compacity 35\%), about 40 are in the outer shell, i.e. $80 \%$ of the NPs offer a fraction of their surface to grafting, which is thus smaller than the total nominal surface of all NPs.
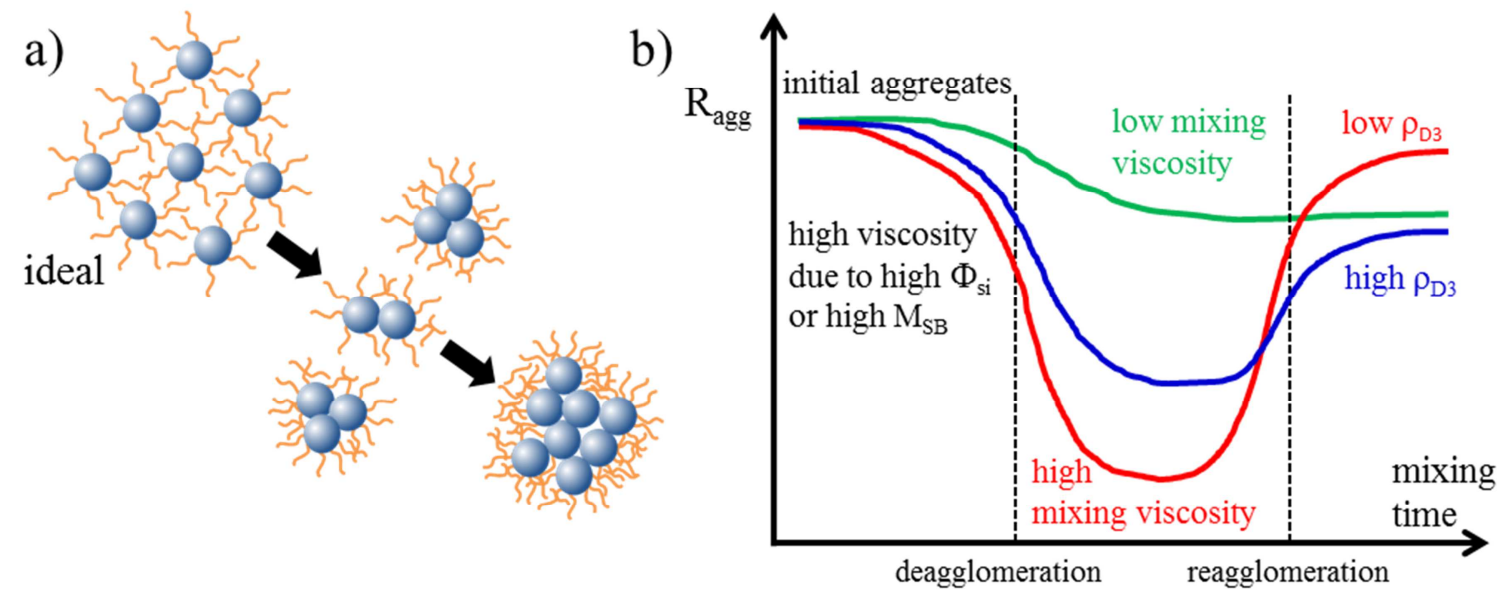

Figure 8: (a) Schematic representation of hypothetical ideally dispersed NPs with nominal grafting density, and the same NPs in experimentally observed aggregates of increasing size with grafting of the same number of molecules only on the aggregate surface. Obviously the aggregate grafting density increases in this agglomeration process. (b) Possible evolution of silica aggregate size during the mixing process allowing a rationalization of our results.

If we approximate for simplicity the surface of an aggregate containing $\mathrm{N}_{\mathrm{agg}}$ silica NPs by the surface of a sphere of radius $\mathrm{R}_{\mathrm{agg}}$, then by conservation of the number of grafts the aggregate grafting density reads:

$$
\rho_{\mathrm{D}_{3}}^{\mathrm{agg}}=\rho_{\mathrm{D}_{3}} \mathrm{~N}_{\mathrm{agg}}\left(\frac{\mathrm{R}_{\mathrm{si}}}{\mathrm{R}_{\mathrm{agg}}}\right)^{2}
$$

The aggregation number increases with the aggregate radius, and following eq.(3) we may write for large enough aggregates: 


$$
\mathrm{N}_{\mathrm{agg}}=\kappa\left(\frac{\mathrm{R}_{\mathrm{agg}}}{\mathrm{R}_{\mathrm{si}}}\right)^{3}
$$

Combining eqs.(5) and (6) gives :

$$
\rho_{\mathrm{D}_{3}}^{\mathrm{agg}}=\rho_{\mathrm{D}_{3}} \kappa \frac{\mathrm{R}_{\mathrm{agg}}}{\mathrm{R}_{\mathrm{si}}}
$$

Thus if the compacity stays approximately constant, typically in the 35\%-range for the samples discussed here (cf. Table 2), then the aggregate grafting density exceeds strongly the nominal NP grafting density for big enough aggregates. Naturally, taking into account more precise variations of $\kappa$, or details of the aggregate surface and internal structure, will fine tune the results, but this is not of importance for the present argument.

The following mechanism is proposed to rationalize the observed evolution of aggregate size, cf. Figures $7 \mathrm{a}$ and $\mathrm{b}$. In the mixing process, due to the action of the torque transmitted by the viscosity set by both chain mass and silica fraction, initial silica aggregates are broken up to smaller ones of typical radius $\mathrm{R}_{\min }$. On these aggregates, some grafting takes place. If the aggregate grafting density exceeds the critical grafting density $\rho_{D 3}^{c}$, then brushes are so dense that these small aggregates are stabilized against re-agglomeration by the grafted brush. If however, the aggregate grafting density is too low to ensure colloidal stability, then re-agglomeration takes place. According to eq.(7), this induces a higher aggregate grafting density because of the lower specific aggregate surface. Once the aggregate grafting density reaches $\rho_{D 3}^{c}$, the process is stopped for the same reasons as before. This process is illustrated in Figure 8. It is thus straightforward to postulate that aggregate radii are set by the grafting density reaching a limiting value:

$$
\rho_{\mathrm{D}_{3}}^{\mathrm{agg}}=\rho_{\mathrm{D}_{3}}^{\mathrm{c}}
$$

Eqs. (7) and (8) can be solved for the aggregate radius as done in eq.(4), where we have also introduced the lower and higher cut-off values, $R_{\min }$ and $R_{\max }$, respectively. The latter are intrinsic to the silica aggregates and the maximum torque in the mixer, and their values cannot be captured by this simple model. To summarize, the critical grafting aggregate density $\rho_{\mathrm{D} 3}{ }^{\mathrm{c}}$ sets the size of the aggregates via the establishment of a dense 
brush regime. Therefore $\rho_{\mathrm{D} 3}{ }^{\mathrm{c}}$ is naturally compared to the onset of the mushroom-tobrush transition.

The data shown in Figure 7 are rather scattered, but clearly a characteristic nominal grafting density $\rho_{\mathrm{D} 3}{ }^{\mathrm{c}}=0.01 \mathrm{~nm}^{-2}$ can be extracted from the decays. This value is quite close to $1 / \mathrm{R}_{\mathrm{g}}{ }^{2}=0.007 \mathrm{~nm}^{-2}$ for $\mathrm{M}_{\mathrm{SB}}=140 \mathrm{~kg} / \mathrm{mol}$, which is the de Gennes estimate of the

onset of the mushroom-to-brush transition ${ }^{30,31}$. Unfortunately, our data are not precise enough to differentiate critical grafting densities for each mass, which are all in the same range. If on the contrary aggregates are too big, in the sense that their aggregate grafting density exceeds $\rho_{\mathrm{D} 3}{ }^{\mathrm{c}}$, then smaller aggregates may be stabilized, and the big ones deagglomerate.

A consequence of this mechanism is seen in Figure 7: once the nominal grafting density exceeds the critical one, even the smallest possible aggregates are covered by a brush. The size evolution thus saturates at the minimum aggregate breakdown radius $\mathrm{R}_{\min }$ set by the viscosity of the sample in the mixing process, which is itself dominated by the silica content.

\section{Conclusion}

In a previous letter ${ }^{24}$, we have shown that twin samples with close nominal grafting density $\rho_{\mathrm{D} 3}$ have also a close silica structure and plateau moduli. In the present article, the dependence of structure and rheology on the nominal grafting density was further explored by varying the matrix chain mass. The plateau moduli were found not to be affected by the chain mass at $9.5 \% \mathrm{v}$ silica, whereas longer chains move the systems away from a melt flow regime. Concerning structure, we have rationalized the impact of all available parameters - silica fraction, grafting density, and chain mass - in terms of a simple model based on the grafting density on the surface of aggregates. First, both for the average aggregate radius and aggregation number, the data indicate that higher silica volume fractions lead to smaller and lighter aggregates, at least at high grafting, presumably due to a better break-down of aggregates at high viscosity in the melt mixing process. In the model, the decrease of the minimum aggregate size is expressed empirically by a smaller plateau value $\mathrm{R}_{\min }$. Note that the comparison with the previously 
observed $\Phi_{\mathrm{si}}$ dependence is not straightforward, due to the simultaneous change of $\Phi_{\mathrm{si}}$ and $\rho_{\mathrm{D} 3}$ (see SI for comparison). Secondly, higher nominal grafting densities induce the stabilization of smaller aggregates, which leads to a decrease of the aggregate radius with the $\rho_{\mathrm{D} 3}$ observed here. Alternatively, lowering the chain mass also increases $\rho_{\mathrm{D} 3}$, with the same effect.

In the framework of our model, this decrease is governed by a critical grafting density $\rho_{\mathrm{D} 3}{ }^{\mathrm{c}}$, which is identical for both silica contents. The critical value $\rho_{\mathrm{D} 3}{ }^{\mathrm{c}}=0.01 \mathrm{~nm}^{-2}$ is found to be compatible with the onset of the mushroom-to-brush transition of the polymer layer grafted on aggregates. For aggregates of small enough radii, the effective aggregate grafting density is below this value and thus too low to stabilize such aggregates: they grow up to a radius where the aggregate grafting density is equal to $\rho_{\mathrm{D} 3}{ }^{\mathrm{c}}$.

We have seen that changing the grafting density $\rho_{\mathrm{D} 3}$ gives us the possibility to tune the filler structure in complex nanocomposites. The combination of chain mass $\mathrm{M}_{\mathrm{SB}}$ and matrix composition \%D3 in eq.(1) allows one then to tune the rheology, as long as the ratio of $\% \mathrm{D} 3$ to $\mathrm{M}_{\mathrm{SB}}$ is kept constant. A high $\% \mathrm{D} 3$, together with a high mass, gives nanocomposites with a long rubbery plateau, and a strongly hindered flow regime. In the opposite situation, a normal flow regime is found, together with a short relaxation time and thus a short rubbery plateau. Finally, our findings suggest that it is possible to tune rheological behavior while keeping the silica structure unchanged, opening original possibilities of rational design of complex nanocomposite systems for applications. We also find encouraging that it is possible to develop fundamental understanding based on a detailed structural model involving both TEM and SAXS in simplified industrial nanocomposites. By construction, these systems still bear a certain model character (e.g., well-defined polymer masses as used here), but are close to applications due to the use of a highly disordered industrial filler. An interesting perspective of the present work would be a structural study of a more polydisperse polymer system, thereby approaching industrial chain distributions, and investigate the effect of the grafting density.

Acknowledgements: We are thankful for a "Chercheur d'Avenir" grant by the Languedoc-Roussillon region (J.O.) and Ph.D. funding "CIFRE” by Michelin (G.P.B.). Beamtime by ESRF (Grenoble, France) is gratefully acknowledged. A few data points in 
Figure 7 are based on SANS measurements performed at JCNS (FRM2, Munich, Germany). DMA measurements have been performed with A. Mujtaba, T. ThurnAlbrecht, and K. Saalwächter (Martin-Luther-University, Halle/Saale, Germany).

\section{References}

1. J. Jancar, J. F. Douglas, F. W. Star,, S. K. Kumar, P. Cassagnau, A. J. Lesser, S. S. Sternstein and M. J. Buehler, Polymer, 2010, 51, 3321-3343.

2. G. Heinrich, M. Kluppel and T. A. Vilgis, Current Opinion in Solid State \& Materials Science, 2002, 6, 195-203.

3. J. E. Mark, B. Erman and F. R. Eirich, Science and Technology of Rubber, Academic Press, San Diego, 1994.

4. G. Schmidt and M. M. Malwitz, Current Opinion in Colloid \& Interface Science, 2003, 8, 103-108.

5. J. Oberdisse, Soft Matter, 2006, 2, 29-36.

6. N. Jouault, P. Vallat, F. Dalmas, S. Said, J. Jestin and F. Boue, Macromolecules, 2009, 42, 2031-2040.

7. N. Jouault, F. Dalmas, F. Boue and J. Jestin, Polymer, 2012, 53, 761-775.

8. G. Capuano, G. Filippone, G. Romeo and D. Acierno, Langmuir, 2012, 28, 54585463.

9. M. I. Aranguren, E. Mora, J. V. Degroot and C. W. Macosko, Journal of Rheology, 1992, 36, 1165-1182.

10. S. S. Choi, B. H. Park and H. Song, Polymers for Advanced Technologies, 2004, 15, 122-127.

11. M. Tatou, A. C. Genix, A. Imaz, J. Forcada, A. Banc, S. R., I. Grillo and J. Oberdisse, Macromolecules, 2011, 44, 9029-9039.

12. L. Conzatti, G. Costa, M. Castellano, A. Turturro, F. M. Negroni and J. F. Gerard, Macromolecular Materials and Engineering, 2008, 293, 178-187.

13. J. Ramier, C. Gauthier, L. Chazeau, L. Stelandre and L. Guy, Journal of Polymer Science Part B-Polymer Physics, 2007, 45, 286-298.

14. D. Sunday, J. Ilavsky and D. L. Green, Macromolecules, 2012, 45, 4007-4011.

15. A. Banc, A. C. Genix, M. Chirat, C. Dupas, S. Caillol, M. Sztucki and J. Oberdisse, accepted in Macromolecules.

16. C. Chevigny, F. Dalmas, E. Di Cola, D. Gigmes, D. Bertin, F. Boué and J. Jestin, Macromolecules, 2011, 44, 122-133. 
17. B. Natarajan, T. Neely, A. Rungta, B. C. Benicewicz and L. S. Schadler, Macromolecules, 2013, 46, 4909-4918.

18. J. H. Maas, G. J. Fleer, F. A. M. Leermakers and M. A. Cohen Stuart, Langmuir, 2002, 18, 8871-8880.

19. S. K. Kumar, N. Jouault, B. Benicewicz and T. Neely, Macromolecules, 2013, 46, 3199-3214.

20. C. Chevigny, J. Jestin, D. Gigmes, R. Schweins, E. Di-Cola, F. Dalmas, D. Bertin and F. Boue, Macromolecules, 2010, 43, 4833-4837.

21. R. Hasegawa, Y. Aoki and M. Doi, Macromolecules, 1996, 29, 6656-6662.

22. G. P. Baeza, A. C. Genix, C. Degrandcourt, L. Petitjean, J. Gummel, M. Couty and J. Oberdisse, Macromolecules, 2013, 46, 317-329.

23. G. P. Baeza, A. C. Genix, C. Degrandcourt, L. Petitjean, J. Gummel, R. Schweins, M. Couty and J. Oberdisse, Macromolecules, 2013, 46, 6388-6394.

24. G. P. Baeza, A. C. Genix, C. Degrandcourt, J. Gummel, A. Mujtaba, K. Saalwachter, T. Thurn-Albrecht, M. Couty and J. Oberdisse, Macro Letters, 2014, 3, 448-452.

25. M. Doi and S. F. Edwards, The Theory of Polymer Dynamics, Oxford University Press, Oxford, 1986.

26. F. Chambon and H. H. Winter, Journal of Rheology, 1987, 31, 683-697.

27. K. Nusser, G. J. Schneider and D. Richter, Soft Matter, 2011, 7, 7988-7991.

28. P. Cassagnau, Polymer, 2008, 49, 2183-2196.

29. C. Chevigny, N. Jouault, F. Dalmas, F. Boue and J. Jestin, Journal of Polymer Science Part B-Polymer Physics, 2011, 49, 781-791.

30. P. G. De Gennes, Macromolecules, 1980, 13, 1069-1075.

31. M. Aubouy, F. Brochard-Wyart and E. Raphael, Macromolecules, 1993, 26, 5885-5889. 